\title{
Coordination polymer glass for bio-inspired photoelectric conversion application
}

Sanjog S. Nagarkar ${ }^{1}$, Satoshi Horike ${ }^{1}$, Tomoya Itakura ${ }^{2}$, Benjamin Le Ouay ${ }^{3}$, Aude Demessence ${ }^{4}$, Masahiko Tsujimoto ${ }^{1}$, Susumu Kitagawa ${ }^{1}$

${ }^{1}$ Institute For Integrated Cell-Material Sciences (WPI-ICeMS), Kyoto University, Kyoto, Japan, ${ }^{2}$ DENSO Corporation, Aichi, Japan,

${ }^{3}$ Department of Synthetic Chemistry and Biological Chemistry, Graduate School of Engineering, Kyoto University, Kyoto, Japan,

${ }^{4}$ Institut de Recherches sur la Catalyse et I'Environnement de Lyon, Université Claude Bernard Lyon 1, Villeurbanne, FRANCE, Lyon, France

E-mail: nagarkar.sanjog.82c@st.kyoto-u.ac.jp

Development of clean and sustainable energy source is current area of an interest for researchers to alleviate ever increasing energy demand of mankind. Earth receives 120000 terawatts of solar energy every year which is far more than the world wide annual requirement of $\sim 15$ terawatts. Thus efficient solar energy harvesting is expected to play key role in future energy supply. Living organisms harvest solar energy and produce chemical energy to power various cellular functions. They create electrochemical proton gradients by light absorption which is then utilized to carry out chemical reactions and store energy (for example ADP to ATP conversion). In principle, these photo-generated electrochemical gradients can be converted to electric currents to produce power. Thus the natural biochemical machinery inspires researchers to design advance light harvesting technology. In photosynthesis, light induced electron transfer process leads to charge separation with consequent formation of electrochemical gradient. Analogues to this protonic solid with optically generated asymmetric proton concentration (electrochromic gradient) are promising candidates for solid-state bio-inspired photo electric conversion system. However, the limited synthetic approaches for orchestration of reversible stimuli responsiveness synergistic with protonic solids precluded the active development in optically switchable protonic solids. Coordination polymers (CPs) are extensively used for clean energy applications in last decade. Recently, melt quenched glass formation in proton conducting CP is reported by our group. The melted phase is available to uniformly integrate stimuli responsive functionalities in to the CPs at moderate temperatures $\left(100-200^{\circ} \mathrm{C}\right.$ ) as against pure organic or inorganic materials. Further, the conspicuous transparency of the melt quenched CP glass is persuasive for optical applications. In present study, we melt functionalised proton conducting CP [ $\mathrm{Zn}(\mathrm{HPO} 4)(\mathrm{H} 2 \mathrm{PO} 4) 2](\mathrm{ImH} 2) 2$ by photo acid molecule to achieve enhanced and optically switchable protonic dynamics in solid-state. A reversible and efficient proton dynamics modulation in doped $\mathrm{CP}$ glass is realized by light irradiation. The proton dynamics in the material was successfully switched between low and high activity states by light irradiation for 8 consecutive cycles. Further, to demonstrate the potential of CP glass for photo electric conversion application a long lived asymmetric proton gradient in functionalized CP glass was developed by light irradiation and characterized by open circuit voltage (OCV) measurement. The detailed insights in to the structure of doped CP glass and optically controllable proton dynamics in CPs glass were gained by X-ray absorption spectroscopy, X-ray total scattering measurements and optical spectroscopy techniques. Recently, the above work is accepted for the publication in Angewandte Chemie International Edition journal.

[1] Xie X. \& Bakker E. (2014). Phys. Chem. Chem. Phys. 16, 19781-19789.

[2] Batten S. R. \& Robson, R. (1998). Angew. Chem. Int. Ed. 37, 1460-1494.

[3] Kitagawa S. et al. (2017). Angew. Chem. Int. Ed. doi: 10.1002/anie.201700962.
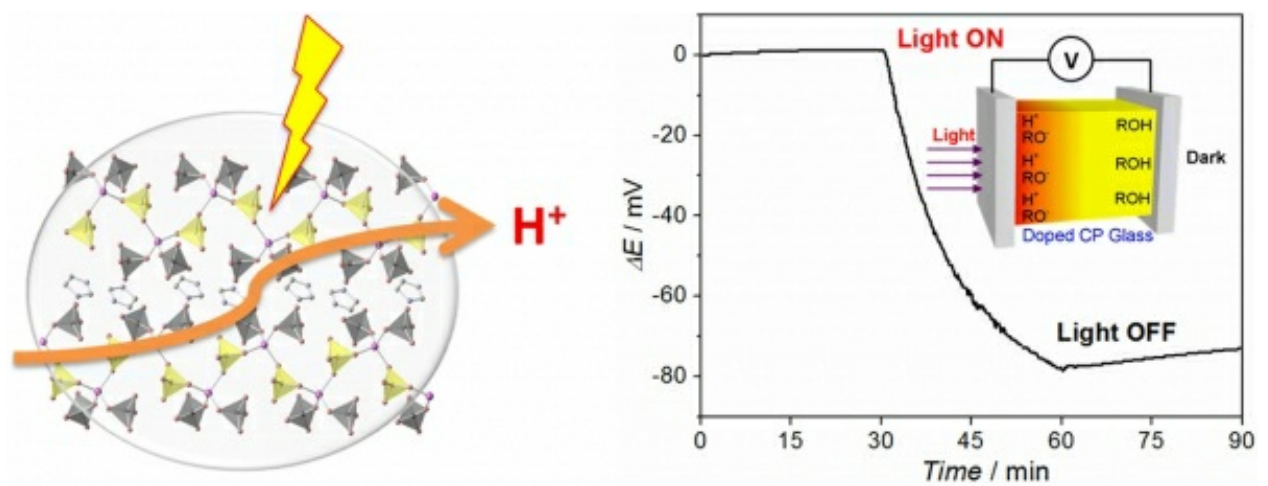

Keywords: photoelectric conversion, coordination polymer, glass 